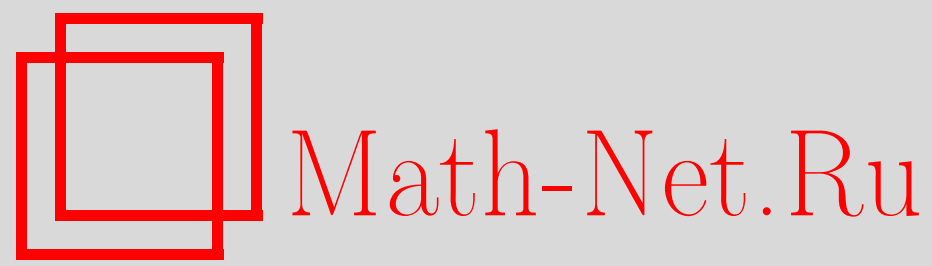

Общероссийский математический портал 
И. А. Мельникова, Свойства морсовской формы, определяющей компактное слоение на $M_{g}^{2}, M a$ тем. заметки, 1996, том 60, выпуск 6, 942-945

DOI: https://doi.org/10.4213/mzm1915

Использование Общероссийского математического портала Math- 
Net.Ru подразумевает, что вы прочитали и согласны с пользовательским соглашением

http://www . mathnet.ru/rus/agreement

Параметры загрузки:

IP : 18.234 .156 .22

26 апреля 2023 г., 08:24:12 


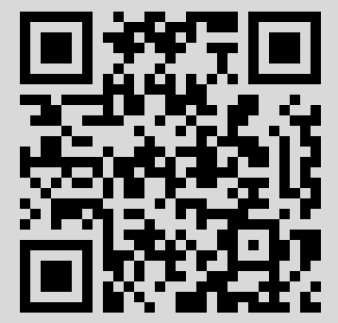




\section{СВОЙСТВА МОРСОВСКОЙ ФОРМЫ, ОПРЕДЕЛЯЮЩЕЙ КОМПАКТНОЕ СЛОЕНИЕ НА $M_{g}^{2}$}

\section{И. А. Мельникова}

П. Арнуа и Г. Левиттом в работах [1], [2] было показано, что топология слоения морсовской формы $\omega$ на компактном многообразии тесно связана со структурой отображения интегрирования $[\omega]: H_{1}(M) \rightarrow \mathbb{R}$. В данной работе рассматривается слоение морсовской формы на двумерном многообразии $M_{g}^{2}$. Изучается связь подгруппы $\operatorname{Ker}[\omega] \subset H_{1}\left(M_{g}^{2}\right)$ с топологией слоения. Получены следующие результаты: рассмотрена структура подгруппы $\operatorname{Ker}[\omega]$ компактного слоения и доказан критерий компактности слоения.

Работа выполнена при частичной поддержке Российского фонда фундаментальных исследований, грант № 96-01-00276.

(C) И.А. МЕльниковА 
1. Предварительные определения. Рассмотрим на многообразии $M_{g}^{2}$ замкнутую форму $\omega$ с морсовскими особенностями. На множестве $M_{g}^{2} \backslash \operatorname{Sing} \omega$ она определяет слоение $\mathscr{F}$.

Определим на многообразии $M_{g}^{2}$ слоение с особенностями $\mathscr{F} \omega$ следующим образом.

Пусть в достаточно малой окрестности особой точки $p \in \operatorname{Sing} \omega$ слоение $\mathscr{F}$ локально определяется уровнями функции $f_{p}$, причем $f_{p}(p)=0$.

ОПРЕДЕЛЕНИЕ 1. Неособим слоем слоения $\mathscr{F} \omega$ назьвается слой $\gamma \in \mathscr{F}$ такой, что $\forall p \in \operatorname{Sing} \omega \gamma \cap f_{p}^{-1}(0)=\varnothing$.

Обозначим $F_{p}=p \cup\left\{\gamma \in \mathscr{F} \mid \gamma \cap f_{p}^{-1}(0) \neq \varnothing\right\}$. Пусть $F=\bigcup_{p \in \operatorname{Sing} \omega} F_{p}$.

ОПРЕДЕЛЕНИЕ 2. Особъм слоем слоения $\mathscr{F} \omega$ назьвается компонента связности множества $F$.

Поскольку форма морсовская, то особых слоев конечное число.

Слоение $\mathscr{F} \omega$ называется компактным, если все его слои компактны.

Замкнутая форма $\omega$ определяет отображение интегрирования по циклам [ $\omega]$ : $H_{1}\left(M_{g}^{2}\right) \rightarrow \mathbb{R}$. Образ $\operatorname{Im}[\omega]$ этого отображения представляет собой группу периодов формы $\omega$. Заметим, что $\operatorname{rk} \operatorname{Im}[\omega]=\operatorname{dirr} \omega+1$, где $\operatorname{dirr} \omega-$ степень иррациональности формы $\omega$.

Если $\operatorname{dirr} \omega \leqslant 0$, то слоение $\mathscr{F} \omega$ компактно [3]. Если $\operatorname{dirr} \omega \geqslant g$, то слоение $\mathscr{F} \omega$ имеет некомпактный слой [4]. Если же $0<\operatorname{dirr} \omega<g$, то слоение может оказаться как компактным, так и некомпактньм. Изучение структуры подгруппы Ker[ $\omega]$ позволяет и в последнем случае получить условия компактности слоения.

Рассмотрим операцию пересечения 1-циклов

$$
\varphi: H_{1}\left(M_{g}^{2}\right) \times H_{1}\left(M_{g}^{2}\right) \rightarrow \mathbb{Z},
$$

являющуюся невырожденным, кососимметрическим билинейным отображением.

Ограничение отображения $\varphi$ на подгруппу $\operatorname{Ker}[\omega] \subset H_{1}\left(M_{g}^{2}\right)$ обозначим через $\varphi_{\omega}$ :

$$
\varphi_{\omega}: \operatorname{Ker}[\omega] \times \operatorname{Ker}[\omega] \rightarrow \mathbb{Z}
$$

Очевидно, $\operatorname{rk} \operatorname{Ker} \varphi_{\omega} \leqslant \operatorname{rk} \operatorname{Ker}[\omega]=2 g-(\operatorname{dirr} \omega+1)$. Для малых значений $\operatorname{dirr} \omega$ существует лучшая оценка.

ПРЕДЛОЖЕНИЕ 1. $\mathrm{rk} \operatorname{Ker} \varphi_{\omega} \leqslant \operatorname{dirr} \omega+1$.

ДокаЗАТЕЛЬСтво. Пусть $\operatorname{Ker} \varphi_{\omega}=\left\langle z_{1}, \ldots, z_{k}\right\rangle$. Обозначим через $D z_{i}$ циклы, двойственные к $z_{i}$, тогда $D z_{i} \circ z_{j}=\delta_{i j}$. Предположим, что $\sum n_{i} \int_{D z_{i}} \omega=0$. Обозначим $z=\sum n_{i} D z_{i}$, тогда, очевидно, $z \in \operatorname{Ker}[\omega]$ и $z \circ z_{j}=n_{j}$. Поскольку $z_{j} \in \operatorname{Ker} \varphi_{\omega}$, то все $n_{j}=0$. Следовательно, интегралы $\int_{D z_{i}} \omega$ линейно независимы над $\mathbb{Q}$ и $\operatorname{dirr} \omega \geqslant k-1$. Предложение доказано.

Подгруппа $H \subset H_{1}\left(M_{g}^{2}\right)$ такая, что $\forall x, y \in H x \circ y=0$, называется uзотponной относительно операции пересечения ииклов. Изотропная подгруппа $H$ назьвается максимальной, если $\forall x \notin H \exists y \in H x \circ y \neq 0$.

Заметим, что подгруппа $\operatorname{Ker} \varphi \omega$ является изотропной.

ПРЕДЛОЖЕНИЕ 2. Пусть $H_{0}$ - максимальная изотропная подәруппа $\boldsymbol{\theta}$ əpynne $\operatorname{Ker}[\omega]$. Тогдa $\operatorname{rk} H_{0}=\frac{1}{2}(\operatorname{rk} \operatorname{Ker}[\omega]+\operatorname{rk} \operatorname{Ker} \varphi \omega)$. 
ДокАЗАТЕЛЬСтво. Поскольку отображение $\varphi_{\omega}$ является симплектической формой на пространстве $\operatorname{Ker}[\omega] \otimes \mathbb{R}$, то утверждение следует из разложения симплектического пространства $\operatorname{Ker}[\omega] \otimes \mathbb{R}$ в прямую сумму попарно ортогональных подпространств: двумерных невырожденных и одномерных вырожденных.

Сопоставим каждому неособому компактному слою $\gamma \in \mathscr{F}_{\omega}$ его гомологический класс $[\gamma]$. Образ множества неособых компактных слоев при этом отображении порождает подгруппу в $H_{1}\left(M_{g}^{2}\right)$. Обозначим ее $H_{\omega}$. Заметим, что подгруппа $H_{\omega}$ является изотропной и $H_{\omega} \subset \operatorname{Ker}[\omega]$.

\section{2. Свойства морсовской формы, определяющей компактное слое- ние.}

ТЕОРема 1. Пусть на многообразии $M_{g}^{2}$ слоение определяется морсовской формой $\omega$. Если слоение $\mathscr{F}_{\omega}$ компактно, то

$$
\operatorname{rk} \operatorname{Ker} \varphi_{\omega}=\operatorname{dirr} \omega+1
$$

ДокАЗАТЕЛЬСтво. Неособые компактные слои слоения $\mathscr{F} \omega$ порождают подгруппу $H_{\omega} \subset H_{1}\left(M_{g}^{2}\right)$. Число особых слоев конечно, обозначим их $\gamma_{1}^{0}, \ldots, \gamma_{k}^{0}$. Рассмотрим вложения $j_{s}: \gamma_{s}^{0} \rightarrow M_{g}^{2}, s=1, \ldots, k$, и индуцированные отображения гомологий $j_{s *}: H_{1}\left(\gamma_{s}^{0}\right) \rightarrow H_{1}\left(M_{g}^{2}\right)$. В каждой группе $H_{1}\left(\gamma_{s}^{0}\right)$ выберем такую максимальную подгруппу $Z_{s}$, чтобы образ $j_{s *} Z_{s} \subset H_{1}\left(M_{g}^{2}\right)$ был изотропной подгруппой.

Рассмотрим подгруппу $H_{0}=\left\langle H_{\omega}, j_{s *} Z_{s}, s=1, \ldots, k\right\rangle$. Очевидно, подгруппа $H_{0}$ является изотропной и $H_{0} \subset \operatorname{Ker}[\omega]$.

Рассмотрим цикл $z \in H_{1}\left(M_{g}^{2}\right)$ такой, что $z \circ H_{0}=0$. Тогда $z \circ H_{\omega}=0$. В работе [5] (см. доказательство теоремы 1.2) было показано, что если слоение $\mathscr{F} \omega$ компактно и $z \circ H_{\omega}=0$, то цикл $z$ реализуется кривой $\alpha \subset \cup \gamma_{s}^{0}$. Будем считать, что $\alpha=\sum \alpha_{s}$, где $\alpha_{s} \subset \gamma_{s}^{0}$. По условию $z \circ j_{s *} Z_{s}=0$ для всех $s$, следовательно, $\left[\alpha_{s}\right] \circ j_{s *} Z_{s}=0$, поскольку при $p \neq s \alpha_{p} \cap \gamma_{0}^{s}=\varnothing$. Так как $\alpha_{s} \subset \gamma_{0}^{s}$, то $\left[\alpha_{s}\right] \in \operatorname{Im} j_{s *}$, следовательно, в силу построения группы $Z_{s}$ получаем, что $\left[\alpha_{s}\right] \in j_{s *} Z_{s}$ и соответственно $z \in H_{0}$.

Таким образом, подгруппа $H_{0}$ является максимальной в группе $H_{1}\left(M_{g}^{2}\right)$. Следовательно, как было показано в работе [5], rk $H_{0}=g$. С другой стороны, $H_{0}$ максимальнав группе $\operatorname{Ker}[\omega]$ и согласно предложению $2 \mathrm{rk} H_{0}=\frac{1}{2}(\operatorname{rk} \operatorname{Ker}[\omega]+\operatorname{rk} \operatorname{Ker} \varphi \omega)$. Поскольку $\operatorname{dirr} \omega=2 g-1-\operatorname{rk} \operatorname{Ker}[\omega]$, то теорема доказана.

ЗАмЕч АниЕ. В обратную сторону теорема неверна, т.е. сушествуют некомпактные слоения, для которых приведенное равенство выполняется.

3. Критерий компактности слоения. В работе [4] было показано, что если существует $g$ гомологически независимых компактных слоев, то все слои компактны. С учетом структуры подгруппы $\operatorname{Ker}[\omega]$ этот признак компактности можно усилить. Рассмотрим пересечение $H_{\omega} \cap \operatorname{Ker} \varphi_{\omega}$.

Теорема 2. Слоение $\mathscr{F} \omega$ компактно тогда и только тогда, когда

$$
\operatorname{rk}\left(H_{\omega} \cap \operatorname{Ker} \varphi \omega\right) \geqslant \operatorname{dirr} \omega
$$


ДоКАЗАТЕЛЬСтво. Если $\operatorname{dirr} \omega \leqslant 0$, то слоение компактно согласно [3]. Пусть $\operatorname{dirr} \omega \geqslant 1$, тогда $k=\operatorname{rk}\left(H_{\omega} \cap \operatorname{Ker} \varphi_{\omega}\right) \geqslant 1$.

Рассмотрим неособые слои $\gamma_{i} \in \mathscr{F}_{\omega}$ такие, что $H_{\omega} \cap \operatorname{Ker} \varphi_{\omega}=\left\langle\left[\gamma_{1}\right], \ldots,\left[\gamma_{k}\right]\right\rangle$. Как было показано в предложении 1 , интегралы $\int_{D\left[\gamma_{i}\right]} \omega$ линейно независимы над $\mathbb{Q}$ и $\operatorname{dirr} \omega \geqslant k-1$. По условию $\operatorname{dirr} \omega \leqslant k$, следовательно, $\operatorname{dirr} \omega$ определяется интегралами по $D\left[\gamma_{1}\right], \ldots, D\left[\gamma_{k}\right]$ и, возможно, по некоторому циклу $z$.

Разрежем многообразие $M_{g}^{2}$ по слоям $\gamma_{i}$. Поскольку они гомологически независимы, в результате получим связное многообразие $M^{\prime}$ с краем. Число компонент связности края равно $2 k$. Ограничение формы $\omega$ на многообразие $M^{\prime}$ обозначим $\omega^{\prime}$. Поскольку циклы $D\left[\gamma_{i}\right]$ при разрезании исчезают, то $\operatorname{dirr} \omega^{\prime} \leqslant 0$.

Заклеим каждую компоненту связности края диском $D_{i}^{2}$, в результате получим многообразие $M_{g-k}^{2}$. Продолжим форму $\omega^{\prime}$ на диски $D_{i}^{2}$ таким образом, чтобы на каждом $D_{i}^{2}$ полученная форма $\omega^{\prime \prime}$ была морсовской и имела одну особую точку типа максимума или минимума. Очевидно, $\operatorname{dirr} \omega^{\prime \prime} \leqslant 0$ и слоение $\mathscr{F}_{\omega^{\prime \prime}}$, является компактным. Следовательно, компактно и слоение $\mathscr{F} \omega$. В одну сторону теорема доказана.

Обратно, пусть слоение $\mathscr{F} \omega$ компактно. Покажем, что тогда $\operatorname{Im}[\omega]=D \operatorname{Ker} \varphi \omega$. Действительно, согласно доказательству предложения $1 D \operatorname{Ker} \varphi_{\omega} \subseteq \operatorname{Im}[\omega]$. По теореме 1 rk Ker $\varphi_{\omega}=\operatorname{dirr} \omega+1$, с другой стороны, $\operatorname{rk} \operatorname{Im}[\omega]=\operatorname{dirr} \omega+1$, следовательно, $\operatorname{Im}[\omega]=D \operatorname{Ker} \varphi \omega$. Таким образом, если слоение $\mathscr{F} \omega$ компактно, то $H_{1}\left(M_{g}^{2}\right)=\operatorname{Ker}[\omega] \oplus D \operatorname{Ker} \varphi \omega$.

Пусть $H_{\omega}=\left\langle\left[\gamma_{1}\right], \ldots,\left[\gamma_{N}\right]\right\rangle$. Из доказательства теоремы 2.2 [5] следует, что $\operatorname{dirr} \omega+1=\operatorname{rk}_{\mathbf{Q}}\left\{\int_{D \gamma_{1}} \omega, \ldots, \int_{D \gamma_{N}} \omega\right\}$. Перенумеруем слои таким образом, что $\forall i \leqslant s \int_{D \gamma_{i}} \omega \neq 0, \mathrm{a} \forall i>s \int_{D \gamma_{i}} \omega=0$. Тогда $\operatorname{dirr} \omega+1=\operatorname{rk}_{\mathbf{Q}}\left\{\int_{D \gamma_{1}} \omega, \ldots, \int_{D \gamma_{s}} \omega\right\} \leqslant \operatorname{rk}\left\langle\left[\gamma_{1}\right], \ldots,\left[\gamma_{s}\right]\right\rangle \leqslant \operatorname{rk}\left(H_{\omega} \cap \operatorname{Ker} \varphi_{w}\right)$, поскольку, как было показано выше, если $\int_{D \gamma_{i}} \omega \neq 0$, то $\left[\gamma_{i}\right] \in \operatorname{Ker} \varphi_{\omega}$. Теорема доказана.

Таким образом, для компактности слоения достаточно существования $\operatorname{dirr} \omega$ гомологически независимых слоев, классы гомологий которых принадлежат подгруппе $\operatorname{Ker} \varphi_{\omega}$. В частности, если $\operatorname{dirr} \omega=1$ и существует негомологичный нулю слой $\gamma,[\gamma] \in \operatorname{Ker} \varphi \omega$, то слоение компактно.

Автор выражает благодарность профессору А. С. Мищенко за внимание к работе и полезные обсуждения.

Московский государственный университет

Поступило им. М.В. Ломоносова 18.06 .96

E-mail: melnikova@micron.msk.ru

\section{СПИСОК ЦИТИРОВАННОЙ ЛИТЕРАТУРЫ}

1. Arnoux P., Levitt G. // Invent. Math. 1986. V. 84. P. 141-156. 2. Levitt G. // Invent. Math. 1987. V. 88. Р. 635-667. 3. Новиков С. П. // УМН. 1982. Т. 37. № 5. С. 3-49. 4. Мельникова И. А. // Матем. заметки. 1993. Т. 53. № 3.

С. 158-160. 5. Мельникова И. А. Компактные слоения морсовских форм. Дисс. ... к. ф..-м. н. М.: МГУ, 1996. 